\title{
Marketing Channel Sustainable Development on Self-organization
}

\author{
Jinfu Wang \\ School of Management, Xi' an Polytechnic University, Xi'an 710048, China \\ E-mail: jinfubest@163.com
}

\begin{abstract}
Marketing channel plays a key role for the success of an enterprise in gaining competitive edge, markets demanding agility and quick market responsiveness which represent complex phenomena in global competition. To discover the behavior and mechanism of organization, nowadays, more and more people have paid attention to the complexity theory such as self-organization, dissipative structure theory, synergetic theory, etc. The paper introduces the concept of self-organization theories and marketing channel firstly. Then enlightened by the systematical methodology, the paper puts forward the concept of sustainable development of organization on the basis of analyzing the characteristics of self-organization marketing channel. A thorough analysis of the mechanism of marketing channel sustainable development has been made accordingly. Finally, the paper adopts the marketing channel of TCL Group as an example, indicating that the success of TCL Group is mostly due to its effective and efficient self-organized marketing channel.
\end{abstract}

Keywords: Complexity phenomenon, Marketing channel, Self-organization, Sustainable development

\section{Introduction}

The globalization of economy with more diversified and frequent communion and an increase in customer expectations make a chaotic and complex environment for enterprises. Success or failure for most enterprises is determined by how effectively and efficiently their products are sold through their marketing channel in economic environment described "Complexity phenomena". But influences of complex factors, problems such as instability and conflict between channel members existed in marketing channel, have become shackles of many enterprises. Given this situation, considerable marketing channel research has focused on interrelationships among an enterprise and its channel members can be managed better. The study points out that agility, reactivity, flexibility and autonomous marketing channel play a key role for the success of an enterprise in gaining competitive edge, markets demanding agility and quick market response in global competition. These are all in accordance with theoretic foundation of self-organization mechanism. There are many articles about self-organization, but most of them focus on supply chain management and application of intra-organization system. To the best of our knowledge, past research has paid little attention to self-organization marketing channel, and empirical studies remain scarce.

Indeed, self-organization mechanism may be more prevalent in highly complex and uncertain environment such as marketing channel than in simple intra-organizational system. In other words, marketing channel may produce greater benefits and effectiveness by using self-organization mechanism.

The objective of this study is to propose a self-organization framework for marketing channel on the basis of the existing theories, and bring forward the concept of marketing channel sustainable development. Firstly, the theoretical rationale and concept will be presented, the characteristics of self-organization marketing channel will be explained next, followed by the methods and mechanism of marketing channel sustainable development, Finally, a case of marketing channel of TCL group is specialized for empirical research.

\section{Concept model of SOMC (Self-organizational Marketing Channel)}

\subsection{Marketing Channel}

Marketing channel can be defined as the set of external organizations that a firm uses to achieve its distribution objectives. Essentially, a channel is the route, a path, or a conduit, through which products or the value of products flow from the manufacturer to the ultimate user. During the process, the channel members compete and cooperate with the external circumstance constantly. Management and marketing researchers alike contend that the environment is in a constant process of change, so the adaptation behavior of marketing channels is very important, which has been well proved in many relevant articles. Specifically, the structural adaptation and strategies of marketing channels to environmental conditions have been shown to be positively related to organizational performance.

In another aspect, there has been a growing interest in the issue of marketing channel focused on the coordination of business activities, which brings the essential of structure of marketing channel and also is concerned with the trust and cooperation relationship among channel members. The objective of this study is to improve the efficiency of 
marketing channel in complex distribution channel environment.

\subsection{Concept of Self-Organization}

Self-organization is a key concept in complexity theory. According to Tharumarajah, self-organization is defined as the ability of an entity (or a system as an entity incorporating a collection of sub-entities) to adapt itself to prevailing conditions of its environment. Self-organization is also known as the relative agility of an entity, which ensures its optimal function through minimum help or intervention from external (e.g. human operator) or internal (e.g. other entity) components of the system. The Intelligent Manufacturing Systems World Project defines self-organized system as a system that is not coordinated by the exterior. Entities are autonomous and execute the tasks together. Through interaction and mutual comprehension, the sum or the combination of individual tasks allows to manage an order, a good or a service which is more global.

Self-organization system must have two aspects. One is spontaneity of internal diversity, the other is instability of internal selectivity in mutual commuting with external environment, maintaining the open state of organization inside or outside. Then the diversified subsystem of organization will compete and cooperate with each other and as a result, a sequence parameter, which coincident with the sequence parameter produced by environment of organization, is produced. The development of self-organization system keeps consistent with the change of environment, which means self-organization has acclimatization.

From the above points, we can view that the associated characteristics of self-organization are (1) autonomous, (2) cooperative, (3) transformative. Term of "autonomous" refers to the possible capability to remain its quality or state without outside control; "cooperative" means to maintain stability relationship among subsystem of inter-organization and with the external environment; "transformative" means the existing independent capability.

\subsection{Conceptual Model of Self-Organization Marketing Channel (SOMC)}

I.Prigogine, the founder of dissipative structures theory and K.J.Arrow, the Nobel economics prize winner put forward that economic activity is a continuous evolutive complexity system. Subsystems in organization check each other as the external strength to promote each function to be carried out: Design function and framework of organization according to business procedure, every link puts forward the requirements to the upper region and is appraised by lower member at the same time, forming a "self-organized system" characterizing "function coupling" with internal key motivity at last.

\subsubsection{The Structure and the Mechanism of Marketing Channel.}

The channel member of traditional marketing channel system have different objectives, which causes the cooperation and scheduling problem in an overwhelming complexity which is often difficult to solve. Even more, flexibility is difficult to achieve in the traditional hierarchical system because of the rigid structure. For this reason, a dynamic approach based on autonomous and explicitly independent systems and is able to evolve in a changing environment is required, and a self-organization and its manner of behavior are emerged in the process of interactions between autonomous entities. This approach gives more flexibility and is more suitable for the modeling and control of complex system. To learn from environment and evolve together with it is the key reason to the survival of the systems in changing environment.

The composition principle of the traditional marketing channel and operation process can be simply summarized and shown as a pursuing structure in figure 1 . Real line means the connections of members. The output of channel is determined by whole organization, and the performance of each member is determined by plan in advance. The connections among members are single and the relationship is stiff. Therefore, CM (channel member) individual is lack of the right of autonomy and the behaviors of member depend on control management of the manufacturer instead of the relationships with other members and external environment condition.

Self-organization requires changing the relationships among channel members and external marketing circumstance. It regards each member as an independent decision-maker, which has the nature of autonomy and can make decision according to one's own interests and environmental change. On the other hand, due to the inseparability of working cost and transaction cost, it is necessary to keep all units as one integrative organization, so as to form an organic whole connected with each other. Dotted line means to set up market relationships, which meets the flexible structure and adaptability to change in the channel organization.

\subsubsection{The Characteristics of Self-Organization Marketing Channel}

This study introduces the concept of self-organization marketing channel so as to make the sustainable development of channel of distribution become reality. The characteristics of the self-organization marketing channel are concluded as follows.

(1) Self-adaptability to the environment. Self-organization system has the ability to reorganize its structure and interactive mode automatically, so as to firm new hierarchical structure and function to adapt to the changing of 158 
environment. There's no fixed system structure and equations but only the advanced learning capacity remained inside the channel of distribution system.

(2) Key order-parameter slaved system evolution - In Haken's synergetic theory, there's a famous conception called "slaving principle", which means there always have some key parameters in the complex system, which are called "order-parameter" and change relatively slowly and manage the whole system evolution. The marketing channel system is open, in the process, inside mechanism arose entropy changing $\left(d_{i} s\right)$ and the relationship between system and external circumstance produces system entropy changing $\left(d_{e} s\right)$, so $d s=d_{i} s+d_{e} s, d_{e} s<0$ in the open channel system and $d_{e} s>d_{i} s$, the result is $d_{s}=d_{i} s+d_{e} s<0$, the system becomes orderly from out of order.

(3) Cooperation - Self-organization normally means the existence of both emergence of individual behavior of entity and upward flow of behavior and information. The marketing channel system is an organic syntheses. Minimal degree of cooperation must exist among distributed system's entities to avoid total disorder, which can be provided by different mechanisms like communication, negotiation... The concept of marketing channel self-organization refers to a variety of distinct systemic attributes, such as: self-creation, self-configuration, self-regulation, self-steering, self-maintenance, self-(re-)production, and at the same time, synergic mechanism make the activity of subsystems consistent with the objective of the marketing channel and the integer behavior will be much better.

(4) Environment consonancy -There is much difference among subsystems of marketing channel intra-organization, which is away from equilibrium. But the effectiveness among them are quite strong, which gather adequate favorable factors for self-renewing in the process of exchanging substance, energy and information with environment, which is away from equilibrium, to enable the development of self-organization marketing channel, which is supposed to be impossible, becomes possible. Many facts prove that objective of sustainable development of the enterprises becomes true when mutual benefits are achieved and friendly relationships with the correlated marketing circumstances are established.

\section{The mechanism of marketing channel sustainable development}

\subsection{Concept of Sustainable Development}

The concept of sustainable development is put forward based on the requirements of adaptability to environment for an organization in cruel competition. It refers to that organization should understand and master the characters of developing and changing environment, so as to make it have acclimatization and forecast the developing tendency of environment accordingly. Organization is required to have ability of self-learning and autonomy. The theory foundation is the synergetic theory of Haken, which has two values: masterdom principle and order-parameter. The mathematic formula is:

$$
\xi_{s}(t)=f_{s}\left[\xi_{u}(t) ; t\right]
$$

Thereinto, $\xi_{s}(t)$ means stable pattern, $\xi_{u}(t)$ means unstable pattern. The formula indicates that unstable pattern dominates stable pattern. $\xi_{s}(t)$ changes with $\xi_{u}(t)$ quickly, so the formula of movement process of system is:

$$
X(s, t)=X_{0}(s)+\sum_{u} \xi_{u}(t) v_{u}(s)+\sum_{s} \xi_{s}(t) v_{s}(s)
$$

$\sum_{s} \xi_{s}(t) v_{s}(s)$ is a gather of stable factors, which have a large number but normally disappear in short time, in the movement process of system. $\sum_{u} \xi_{u}(t) v_{u}(s)$ is a gather of unstable factors, which are few and change slowly, in the movement process of system. It keeps system away from equilibrium point, break up the old equilibrium point and establish a new one, pass the unstable point and form another new ordered state. $\xi_{u}$ is called order-parameter. In this process, the system is restructured by itself, which makes the organization harmonize with the changing environment further. Harmonized relationship formed between organization and environment has mutual benefits, which makes the circumstance become reliable and stable for sustainable development of organization.

\subsection{The Sustainable Development of Marketing Channel-Mechanism of Self-Organization}

According to I. Prigogine, It is fluctuation that makes the system away from instable state in the area near equation where system has the ability to anti-jamming. Departure deduced by fluctuation will disappear through self-attenuation and the stability of system will be recurred after interference. The negative feedback mechanism attenuates the fluctuation, as a result, the system remains in the original ordered stable structure and structure-function is optimized accordingly. 
Away from equilibrium, in Nonlinear Dynamical Systems, the system is in a unstable stationary state. Some little stochastic fluctuation may be magnified through interactional activities and bring gigantic fluctuation of macrocosm. So the system will get new ordered state from instable state. When environment change strongly, the positive feedback mechanism will magnify the fluctuation and realize the transformation of different macro-stability states, so as to improve the adaptability of system to environment.

The relationship between channel members is solid reticulate relationship. Each member forms its own ordered structure in the process of competition, cooperation and self-organization showed in figure 2.

\section{The empirical study_Marketing channel of TCL GROUP}

TCL Group is one of the famous corporations in our country specialized in manufacturing and distribution of home electric appliances for over 20 styles and 1,000 varieties. At present, TCL Group has more than 32 sales branch companies, more than 200 business departments and 400 selling centers all over the country. The group starts to implement its global strategy through merging a foreign company successfully. Recent years, it is also succeeded in IT industry such as mobile phone. The above successes are all due to the efficient marketing channel. And more and more people have paid attention to the reforms on marketing channel in TCL frequently.

The high-efficient marketing channel is the key factor for the TCL group to achieve leading position in many areas. And also the channel has reflected the requirements of $3 \mathrm{C}$, which are management centralization, data concentration and apply composition. Different organizations and departments can exchange information rapidly. Besides all the channel members have same objective, the typical characteristic of those members is localization and unanimity.

Because TCL has integrative order management, visual physical data and intelligent management of supplementary goods, information is transferred effectively among channel members by organizing orders, stock, transportation and plans orderly. The characteristics of the marketing channel of TCL can be concluded in the structure showed in figure 3.

1) Integrative order flow: In the process of order flow from end user to manufacturer, each channel member, including sales department, region delivery center and 3PL, can manage the order on the same administration platform. So the relationship between subsystems in channel is harmonious and the cost of the channel is reduced accordingly.

2) Logistic information is visual: Through integrated organizing system of logistics, manufacturer and administration department will know the logistic state in each phase, including the present state of the goods and the node products is processed, so the operation of logistics will be improved greatly.

3) Intelligent management for supplementary of goods: integrative logistic information platform with effective cooperation between channel members make supplementary of goods is executed intelligently. Each member can estimate and forecast the quantity and frequency of demands for the goods through relevant model. This mechanism reduces the stock in the forepart of the marketing channel without lowering the quality of services and controls the OOS (Out of stocks) effectively at the same time.

The effective mechanism of marketing channel in TCL achieves a series of distinct effects: Terminal stock is reduced $40 \%$ and the cost of stock is greatly decreased. The quality of the services is improved greatly, especially in delivery. Figures point that, $85 \%$ clients receive the goods within 24 hours, $10 \%$ clients within 36 hours, and 5\% clients within 48 hours. Quick and accurate delivery brings good social image to TCL, therefore, the finance and business situation of TCL Group is improved greatly. So, in a word, the efficient marketing channel is the key important factor to the success of TCL Group.

\section{Conclusions}

The marketing channel plays a key role in the development of enterprises, but the disorder, complex environment, and diversified objects in traditional channel members make the channel inefficient. Lots of studies analyze cooperation and trust between channel members in order to improve the efficiency. The complexity theory includes self-organization and dissipative structure theory, which are applied to the management and economic science so as to bring new method for solving the problem existed in marketing channel, such as conflict. Through the interaction of the entities, self-organization behavior is firmed and enterprise becomes prosperous in disorder. Self-organization of marketing channel provides flexibility and agility in responding to customer demand shifts. The fundamental premise of this theory is the synchronization represented in multiple autonomous business entities. The paper introduces the self-organization theory into the marketing channel, setting up the concept model of self-organization of marketing channel and concept of marketing channel sustainable development. Its mechanism is developed in the process of simulating the self-organization behavior of marketing channel. It puts forward a new methodology for the management of marketing channel. A paradigm is illustrated to show a successful change from the traditional 
manufacturing control and planning to a new self-organization. Finally, an empirical study is given to prove the concept model of self-organization of marketing channel sustainable development.

Se1f-organization principle is one of important theories in system theory domain, which will become a main methodology in the study of management science, whereas it is always thought to be a little far from the view of application. The paper introduces the new concept model of self-organization of marketing channel, which has both theoretical and practical meaning and is tested by the example of TCL group, therefore, develops a new mechanism of marketing channel sustainable development. In addition, more application research will be done after this to test the concept model further, so as to use it in a wider area.

\section{References}

A. Thanurnarajah. (1998). A Self-Organizing Model for Scheduling Distributed Autonomous Manufacturing Systems. Cybernetics \& Systems ISSN 0196-9722. Volume 29, N.5. Pp. 461-480.

Camazine, S, et al. (2001). Self Organization in Biological Systems, Princeton University Press, Princeton, U.S.A.

H. Haken. (1978). Synergetics, Springer, Berlin.

H. Ulrich and G. J. B. (1984). Probst edited, Self-organization and Management of Social Systems, Insights, Promises, Doubts, and Questions, Springer - Verlag.

J. Lorigny, Les. (1992). Systems Autonomes. Dunod, Paris.

Kumar, N. (1996). The power of trust in manufacturer-retailer relationships. Harvard Business Review 74 November-December, pp. 92-106.

Pierre Massotte, Roland Bataille. (2000). Future production systems: Influence of self-organization on approaches to quality engineering. International Journal of Production Economics 64, pp.359-377

Richard T Pascale. (1999). Surfing the edge of chaos. MIT Sloan Management Review. vol.40, 3, Spring, pp.83-94.

Tom Peters. (1988). Thriving on ChaosHandbool for Management Revolution. New York: Alfred A. Knopf.

Zhang Lei, et al. Self-organization simulation and output analysis. IEEE 2000, pp.603-608

Zhang, Wenbin, Pu, Yun. (2004). Supply Chain Management on Self-Organization. Journal of Transportation Engineering and Information, No.1. Vo1.2. Mar.

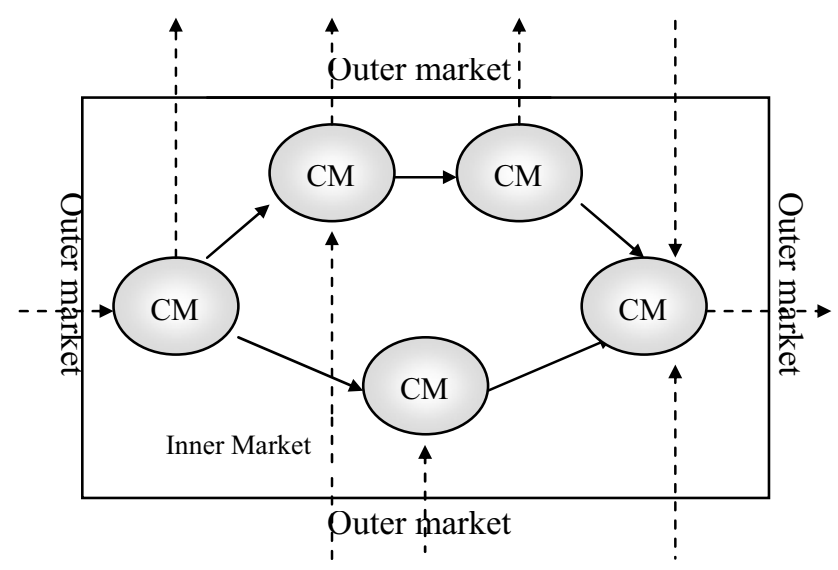

Figure 1. Concept Model of Marketing Channel 


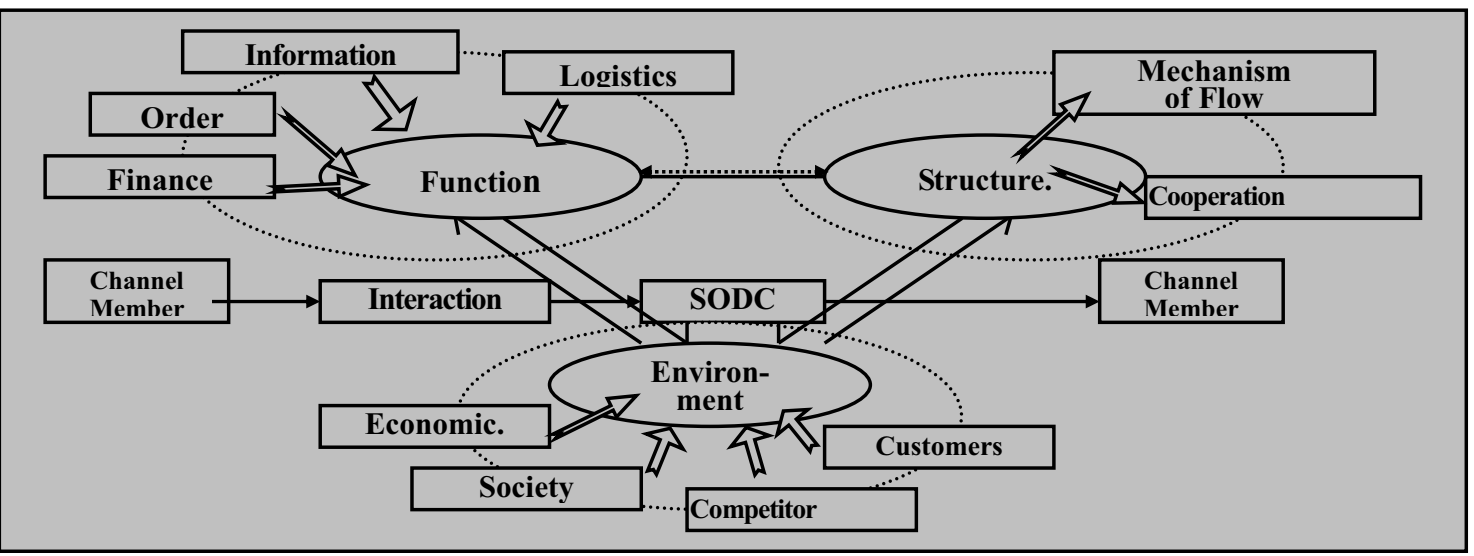

Figure 2. The Mechanism of Self-Organization of Marketing Channel

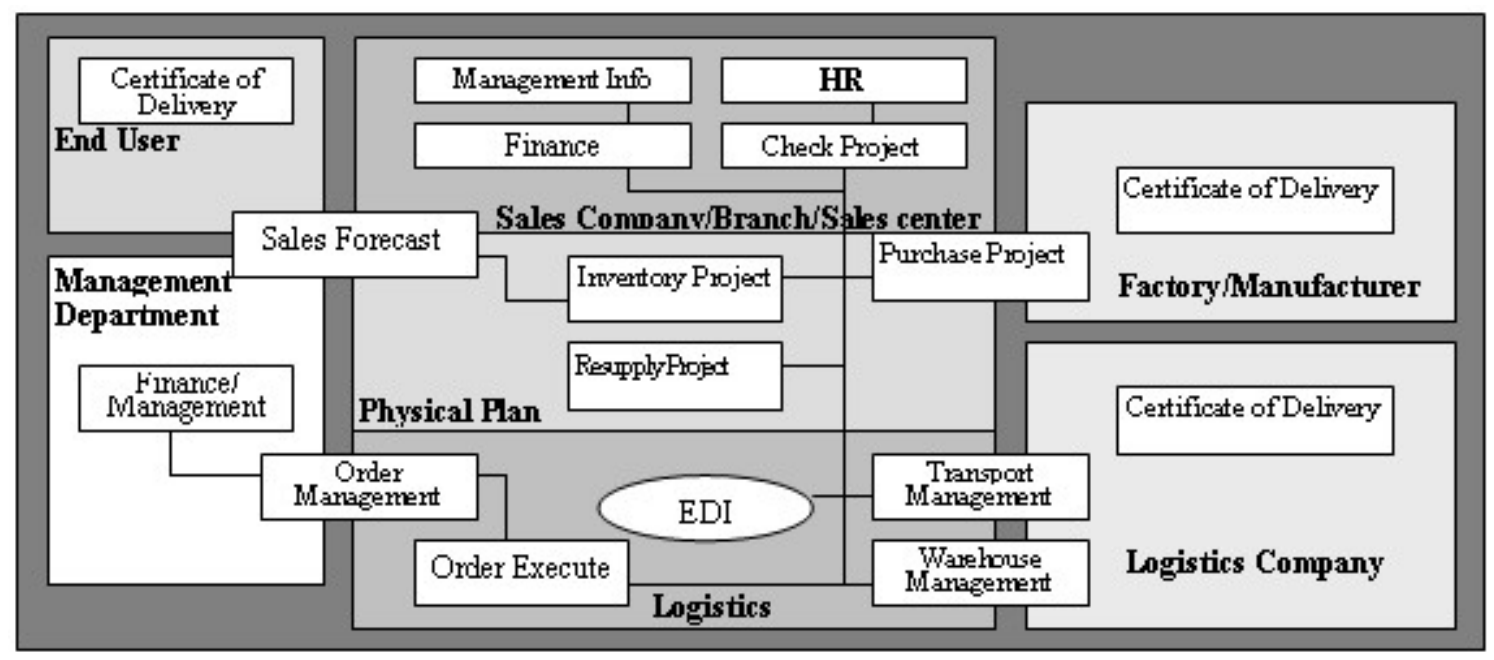

Figure 3. Marketing Channel of TCL Group 